\title{
FINITE DIMENSIONAL INSEPARABLE ALGEBRAS
}

\author{
BY \\ SHUEN YUAN
}

\begin{abstract}
We determine the structure of finite dimensional algebras which are differentiably simple with respect to a set of higher derivations.
\end{abstract}

Let $C$ be a commutative ring of prime characteristic $p$, and let $A$ be a subring of $C$ both with the same identity. By a $p$-basis $\mathfrak{b}$ of $C$ over $A$ we mean a finite subset of $C$ such that for each $t \in \mathfrak{b}, t^{p^{e(t)}} \in A$ for some positive integer $e(t)$ and the set of all monomials $\prod_{t \in \mathfrak{b}} t^{i(t)}, 0 \leqq i(t)<p^{e(t)}$, form an $A$-module basis for $C$. In this note we show that if $A$ is the kernel of a set $\mathrm{g}$ of higher derivations of $C$ such that $C$ is finitely generated as $A$-module and no ideal in $C$, except 0 and 1 , is stable under $\mathrm{g}$, then $C$ admits a $p$-basis over $A$ which must be a field with $\operatorname{Hom}_{A}(C, C)$ $=C[\mathrm{~g}]$. Conversely if $C$ admits a $p$-basis over a field $A$, we show that there is a higher derivation $D$ on $C$ with $\operatorname{Hom}_{A}(C, C)=C[D]$. So no nontrivial ideal can be stable under $D$. When $\mathfrak{g}$ is a set of ordinary derivations, the first statement is given in [4] and is essentially due to Harper [0]. When $C$ is a field, these reduce to results of Sweedler [2] and Weisfeld [3]. We begin this paper with a construction of $p$-basis for local algebras of finite type.

All rings in the following are assumed to be commutative with 1 and of prime characteristic $p$. All modules and ring-homomorphisms are unitary. If $C$ is an $A$-algebra, the structural map $A \rightarrow C$ is assumed to be one-to-one.

1. p-generators. For simplicity of notations, given a subset $X$ of a ring $Y$ we denote by $\mathfrak{F}^{i}(X)$ the subset $\left\{x^{p^{i}} \mid x \in X\right\}$ of $Y$.

Now let $C$ be a local ring with $Q$ as its maximal ideal. Let $E$ be a $C$-algebra such that for some finitely generated nilpotent ideal $J$ in $E, E=C+J$ as a $C$ module direct sum. Let $e=e(J)$ be the least integer such that $\mathfrak{F}^{e+1}(J)=0$. Let $\mathfrak{b}_{e}$ be a subset of $\mathfrak{F}^{e}(J)$ such that $\left\{t+\mathfrak{F}^{e}((J+Q) J) \mid t \in \mathfrak{b}_{e}\right\}$ form a basis for $\mathfrak{F}^{e}(J) / \mathfrak{F}^{e}((J+Q) J)$ over the field $\mathfrak{F}^{e}(C) / \mathfrak{F}^{e}(Q) \cong \mathfrak{F}^{e}(C / Q)$. For each $i$, we are going to construct a subset $\mathfrak{b}_{i}$ of $\mathfrak{F}^{i}(J)$ with the property that

(i) $\left\{t+\mathfrak{F}^{i}((J+Q) J) \mid t \in \mathfrak{b}_{i}\right\}$ form a basis for $\mathfrak{F}^{i}(J) / \mathfrak{F}^{i}((J+Q) J)$ over $\mathfrak{F}^{i}(C) / \mathfrak{F}^{i}(Q)$;

(ii) $\mathfrak{b}_{i+1}=\left\{t^{p} \mid t \in \mathfrak{b}_{i}\right.$ and $\left.t^{p} \neq 0\right\}, 0 \leqq i<e$.

Assume we have already constructed $\mathfrak{b}_{i+1}$. Let $\mathfrak{b}_{i}^{\prime}$ be a subset of $\mathfrak{F}^{i}(J)$ such that for all $t \in \mathfrak{b}_{i+1}, \mathfrak{b}_{i}^{\prime}$ and $\left\{x \in \mathfrak{F}^{i}(J) \mid x^{p}=t\right\}$ has exactly one element in common.

Received by the editors September 16, 1968.

AMS Subject Classifications. Primary 1350, 1360.

Key Words and Phrases. Inseparable algebra, higher derivation, $p$-generators, $p$-basis.

Copyright (C) 1970, American Mathematical Society 
Let $\mathfrak{b}_{i}^{\prime \prime}$ be a subset of $\left\{x \in \mathfrak{F}^{i}(J) \mid x^{p}=0\right\}$ such that the residue classes $t+\mathfrak{F}^{i}((J+Q) J)$, $t \in \mathfrak{b}_{i}^{\prime \prime}$, form a basis for $\left\{x+\mathfrak{F}^{i}((J+Q) J) \mid x \in \mathfrak{F}^{i}(J)\right.$ and $\left.x^{p}=0\right\}$ over $\mathfrak{F}^{i}(C) / \mathfrak{F}^{i}(Q)$. Since the monomials in $\mathfrak{b}_{i+1}$ form a set of generators for the $\mathfrak{F}^{i+1}(C)$-module $\mathfrak{F}^{i+1}(J)$, given $u \in \mathfrak{F}^{i}(J)$ there is a polynomial $\varphi=\varphi\left(\mathfrak{b}_{i}^{\prime}, C_{i}\right)$ in $\mathfrak{b}_{i \text {. with coefficients }}^{\prime}$ in $C_{i}, \varphi$ having no constant term, such that $(u-\varphi)^{p}=0$. It follows from $u=(u-\varphi)$ $+\varphi$ that the set $\mathfrak{b}_{i}=\mathfrak{b}_{i}^{\prime} \cup \mathfrak{b}_{i}^{\prime \prime}$ meets all our requirements.

Hereafter $\mathfrak{b}=\mathfrak{b}_{0}$ will be called a set of $p$-generators for the decomposition $E=C+J$.

We recall that given an algebra $X$ over a ring $Y$, for any $x$ in $X$, the exponent of $x$ is the least nonnegative integer $e(x)$ such that $x^{p e(x)}$ is in $Y$. The exponent of $X$ over $Y$ is the maximum of $\{e(x) \mid x \in X\}$.

EXAmple. Let $C$ be a local $A$-algebra of finite exponent $e$ such that the $A$ module $C$ is finitely generated and flat. Put $E=C \otimes_{A} C$. Then $E=C \otimes 1+J$ where $J=(C \otimes 1) \cdot\{1 \otimes x-x \otimes 1 \mid x \in C\}$. We may assume that the elements of $\mathfrak{b}$ are of the form $1 \otimes t-t \otimes 1$. From $E=(C \otimes 1)[\mathfrak{b}]$ it follows that the inclusion map $C \otimes_{A} A[\{t \mid 1 \otimes t-t \otimes 1 \in \mathfrak{b}\}] \rightarrow E$ is onto. And so the inclusion map $A[\{t \mid 1 \otimes t-t \otimes 1 \in \mathfrak{b}\}] \rightarrow C$ is onto because $C$ over $A$ is actually faithfully flat. In other words, the monomials $\prod t^{i}, 1 \otimes t-t \otimes 1 \in \mathfrak{b}, 0 \leqq i<p^{e(t)}$, form a set of generators for the $A$-module $C$.

2. Higher derivations. By a higher derivation $D$ of rank $\rho, 0<\rho<\infty$, on a ring $C$ we mean a sequence of maps

making the map

$$
D^{(i)}: C \rightarrow C, \quad 1 \leqq i \leqq \rho,
$$

$$
\begin{gathered}
\varphi_{D}: C \rightarrow C[t] /\left(t^{\rho+1}\right), \\
x \rightarrow x+\left(D^{(1)} x\right) t+\cdots+\left(D^{(\rho)} x\right) t^{\rho}
\end{gathered}
$$

a ring-homomorphism. The kernel of $D$ is the set

$$
\left\{x \in C \mid \varphi_{D}(x)=x\right\}=\bigcap\left\{\text { kernel } D^{(i)} \mid 1 \leqq i \leqq \rho\right\} .
$$

Given a set $\mathfrak{g}$ of higher derivations on $C$, we shall denote by $\mathfrak{m}(\mathfrak{g})$ the set of all monomials $\mu$ of the form $D_{1}^{\left(l_{1}\right)} \ldots D_{s}^{\left(l_{s}\right)}, D_{i} \in \mathfrak{g}, 0 \leqq l_{i} \leqq \operatorname{rank} D_{i}$, where $D_{i}^{(0)}$ as usual is understood to be the identity map on $C$. The degree of $\mu$ is the sum $l_{1}+\cdots$ $+l_{s}$. The kernel of $\mathfrak{g}$ is the set $\bigcap\{\operatorname{kernel} D \mid D \in \mathfrak{g}\}$. An ideal $\mathfrak{a}$ in $C$ is said to be stable under $\mathfrak{g}$ if $\mu(\mathfrak{a}) \subset \mathfrak{a}$ for all $\mu$ in $\mathfrak{m}(\mathfrak{g})$.

LeMma 2.1. Let $\mathfrak{g}$ be a set of higher derivations on a ring $C$. Write $A=k e r n e l ~ g$.

(a) $A$ is a subring of $C$ and any idempotent element in $C$ belongs to $A$.

(b) If $C$ has no ideal, except 0 and 1 , stable under $\mathrm{g}$, then $A$ is a field.

(c) If $A$ is a field and if the vector space dimension of $C$ over $A$ is finite, so is the exponent of $C$ over $A$.

(d) If there is a positive integer $\alpha$ such that $p^{\alpha}>\max \{\operatorname{rank} D \mid D \in \mathfrak{g}\}$, then the exponent of $C$ over $A$ is not greater than $\alpha$. 
(e) If $A$ is a field and the exponent of $C$ over $A$ is $\alpha<\infty$, then $x^{p^{\alpha}}=0$ for all nonunit $x$ in $C$.

Proof. (a) Given a pair of ring-homomorphisms

$$
R \underset{v}{\stackrel{u}{\longrightarrow}} S,
$$

it is clear that $\{x \in R \mid u(x)=v(x)\}$ form a subring of $R$. In particular for any higher derivation $D$ on $C$, kernel $D=\left\{x \in C \mid \varphi_{D} x=x\right\}$ form a subring of $C$. So $A$ is a subring of $C$.

Let $D=\left(D^{(1)}, D^{(2)}, \ldots, D^{(l)}, \ldots\right)$ be a higher derivation on $C$. From

$$
\sum_{l}\left(D^{(l)} x^{p}\right) t^{l}=\varphi_{D}\left(x^{p}\right)=\varphi_{D}(x)^{p}=\sum_{i}\left(D^{(i)} x\right)^{p} t^{p i}
$$

we get

$$
\begin{aligned}
D^{(l)}\left(x^{p}\right) & =0, \quad \text { if } l \neq 0(p), \\
D^{(i p)}\left(x^{p}\right) & =D^{(i)}(x)^{p} .
\end{aligned}
$$

Now given a positive integer $l$, we may write $l=q p^{r}$ with $q$ relatively prime to $p$. If $\varepsilon$ is any idempotent in $C$, then

$$
D^{(l)}(\varepsilon)=D^{\left(q p^{r}\right)}\left(\varepsilon^{p^{r+1}}\right)=\left(D^{(q)}\left(\varepsilon^{p}\right)\right)^{p r}=0 .
$$

So $\varepsilon$ belongs to $A$.

(b) For any $x \neq 0$ in $A$ and any $y$ in $C$ we have $\varphi_{D}(x y)=x \varphi_{D}(y), D \in g$. So the ideal $x C$ is stable under $g$ because $D^{(l)}(x y)=x\left(D^{(l)} y\right)$. So $x C=C$ and $x$ is a unit in $C$. From $\varphi_{D}\left(x^{-1}\right)=\varphi_{D}(x)^{-1}=x^{-1}$ it follows that $x^{-1}$ is also in $A$. Hence $A$ must be a field.

(c) Let $C_{i}$ denote the $A$-subalgebra of $C$ generated by $x^{p t}, x \in C$. We have $C_{i+1} \subset C_{i} \subset$ kernel $D^{(l)}$ for all $l \neq 0\left(p^{i}\right)$. In particular the intersection of all $C_{i}$, $i=0,1,2, \ldots$, is contained in $A$. Since $C$ is finite dimensional over $A$, there exists a positive integer $\alpha$ such that $C_{\alpha}=C_{\alpha+1}=C_{\alpha+2}=\cdots$. The exponent of $C$ over $A$ is therefore at most $\alpha$ because $C_{\alpha}$ is contained in $A$.

(d) For any $x$ in $C, D^{(l)}\left(x^{p^{\alpha}}\right)$ is zero for all $D$ in $g$. So the exponent is at most $\alpha$.

(e) For any nonunit $x$ in $C, x^{p^{\alpha}}$ as a nonunit in the field $A$ must be zero.

LEMMA 2.2. Let $\mathrm{g}$ be a set of higher derivations on a local ring $C$. Then the following two statements are equivalent.

(i) No ideal in $C$, except 0 and 1 , is stable under $\mathrm{g}$.

(ii) Given any nonunit $x \neq 0$ in $C$ there is some $\mu \in \mathfrak{m}(\mathfrak{g})$ such that $\mu(x)$ is a unit in $C$.

Proof. (i) $\rightarrow$ (ii). Let $a$ be the nonzero ideal in $C$ generated by $\{\mu(x) \mid \mu \in \mathfrak{m}(\mathfrak{g})\}$ which is clearly stable under $\mathfrak{g}$. So $\mathfrak{a}=C$ and one of the $\mu(x)$ 's must be a unit because $C$ is a local ring. (ii) $\rightarrow$ (i) is trivial. 
If $\mathfrak{g}$ is a set of higher derivations on a ring $C$ with $A=$ kernel $\mathfrak{g}$, then for any $D=\left\{D^{(1)}, \ldots, D^{(\rho)}\right\}$ in $g$, both $1 \otimes D=\left\{1 \otimes D^{(1)}, \ldots, 1 \otimes D^{(\rho)}\right\}$ and $D \otimes 1$ $=\left\{D^{(1)} \otimes 1, \ldots, D^{(0)} \otimes 1\right\}$ are higher derivations on $C \otimes_{A} C$. Let $\mathfrak{g} \otimes \mathfrak{g}$ denote the set of all $1 \otimes D, D \otimes 1$ with $D \in \mathfrak{g}$. We have the following.

Lemma 2.3. Let $\mathrm{g}$ be a set of higher derivations on a ring $C$ with kernel $\mathrm{g}=A$. Assume no ideal in $C$, except 0 and 1 , is stable under $\mathrm{g}$. Then no ideal in $E=C \otimes_{A} C$, except 0 and 1 , is stable under $\mathfrak{g} \otimes \mathfrak{g}$. The kernel of $\mathfrak{g} \otimes \mathfrak{g}$ is equal to $A$.

Proof. We have an exact sequence

$$
0 \longrightarrow A \longrightarrow C \underset{\left.{ }_{(D)}^{(l)}\right)}{\longrightarrow} \amalg C .
$$

Tensoring over $A$ with $C$ we get the exactness of

$$
0 \longrightarrow A \otimes_{A} C \longrightarrow E \frac{}{\left(D^{(l)} \otimes 1\right)} \amalg . E .
$$

This shows kernel $\mathfrak{g} \otimes \mathfrak{g}=\left(A \otimes_{A} C\right) \cap\left(C \otimes_{A} A\right)=A$.

Now assume $\mathfrak{a}$ is a nonzero ideal in $E$ which is stable under $\mathfrak{g} \otimes \mathfrak{g}$ and $\mathfrak{a} \varsubsetneqq E$. Let $\sigma=x_{1} \otimes y_{1}+\cdots+x_{r} \otimes y_{r} \neq 0$ be an element of $\mathfrak{a}$ with $r$ minimal. Clearly $r>1$. Let $\mu \in \mathfrak{m}(\mathfrak{g})$ such that $\mu\left(x_{1}\right)$ is a unit in $C$. The element

$$
(\mu \otimes 1) \sigma=\mu\left(x_{1}\right) \otimes y_{1}+\cdots+\mu\left(x_{r}\right) \otimes y_{r}
$$

cannot be zero because $y_{1}, \ldots, y_{r}$ are linearly independent over the field $A$. Put $\sigma^{\prime}=1 \otimes y_{1}+x_{2}^{\prime} \otimes y_{2}+\cdots+x_{r}^{\prime} \otimes y_{r}$ where $x_{i}^{\prime}=\mu\left(x_{1}\right)^{-1} \mu\left(x_{i}\right)$. Since $\sigma^{\prime} \in \mathfrak{a}$, it cannot belong to $A \otimes_{A} C$ otherwise $r$ would be equal to 1 and we would get a contradiction. So $\left(D^{(l)} \otimes 1\right) \sigma^{\prime}=\left(D^{(l)} x_{2}^{\prime}\right) \otimes y_{2}+\cdots+\left(D^{(l)} x_{r}^{\prime}\right) \otimes y_{r}$ is nonzero for some $D^{(l)}, D \in \mathfrak{g}$. We therefore get a contradiction to the minimality of $r$ because $0 \neq$ $\left(D^{(l)} \otimes 1\right) \sigma^{\prime} \in \mathfrak{a}$. So no nontrivial ideal in $E$ is stable under $\mathfrak{g}$. This completes the proof of the lemma.

THEOREM 2.4. Let $\mathrm{g}$ be a set of higher derivations on a ring $C$ with $A=k e r n e l \mathrm{~g}$. Assume $C$ is finitely generated as an A-module and no ideal in C, except 0 and 1 , is stable under $\mathrm{g}$. Then $C$ admits a p-basis over $A$.

Proof. Since $A$ is a field and $C$ is finite dimensional over $A$, by Lemma 2.1, $C$ is a local ring with nilpotent maximal ideal $Q$. Put

$$
E=C \otimes_{A} C, \quad J=\{1 \otimes x-x \otimes 1 \mid x \in C\} E .
$$

Let $\left\{1 \otimes x_{1}-x_{1} \otimes 1, \ldots, 1 \otimes x_{n}-x_{n} \otimes 1\right\}$ be a set of $p$-generators for $E=$ $C \otimes 1+J$. We claim that $x_{1}, \ldots, x_{n}$ form a $p$-basis for $C$ over $A$. Let $F$ be a subfield of $C$ such that $C=F+Q$. Let $\left\{y_{1}, \ldots, y_{m}\right\}$ be a set of $p$-generators for $C=F+Q$. It is clear that $\left\{y_{1} \otimes 1, \ldots, y_{m} \otimes 1,1 \otimes x_{1}-x_{1} \otimes 1, \ldots, 1 \otimes x_{n}-x_{n} \otimes 1\right\}$ form a set of $p$-generators for $E=F \otimes 1+(Q \otimes 1+J)$. Moreover, by a lemma to be established later,

$$
\prod_{i=1}^{m}\left(y_{i}^{p p_{i}-1} \otimes 1\right) \cdot \prod_{i=1}^{n}\left(1 \otimes x_{i}-x_{i} \otimes 1\right)^{p_{i}-1} \neq 0
$$


where $e_{i}$ (respectively $f_{i}$ ) is the exponent of $1 \otimes x_{i}-x_{i} \otimes 1$ (respectively $y_{i}$ ). It follows that for any $y \in C$,

$$
(y \otimes 1) \prod_{i=1}^{n}\left(1 \otimes x_{i}-x_{i} \otimes 1\right)^{p e_{i}-1}=0
$$

implies $y=0$. So $\left\{1 \otimes x_{1}-x_{1} \otimes 1, \ldots, 1 \otimes x_{n}-x_{n} \otimes 1\right\}$ form a $p$-basis for $E$ over $C \otimes 1$. Now from the binomial expansion of

$$
\left(\left(1 \otimes x_{i}-x_{i} \otimes 1\right)+x_{i} \otimes 1\right)^{d_{i}}=1 \otimes x_{i}^{d_{i}},
$$

it follows that $1 \otimes \prod_{i=1}^{n} x_{i}^{d_{i}}$ can be expressed as a polynomial in

$$
\left\{1 \otimes x_{i}-x_{i} \otimes 1 \mid 1 \leqq i \leqq n\right\}
$$

with coefficients in $C \otimes 1$ and with $\prod_{i=1}^{n}\left(1 \otimes x_{i}-x_{i} \otimes 1\right)^{d_{i}}$ as its highest degree term. This implies that $\left\{\prod_{i=1}^{n} x_{i}^{d_{i}} \mid 0 \leqq d_{i}<p^{e_{i}}\right\}$ is linearly independent over $A$. Since the dimension of $C$ over $A$ is equal to the dimension of $E$ over $C \otimes 1$, $\left\{x_{1}, \ldots, x_{n}\right\}$ must be a $p$-basis for $C$ over $A$.

COROLlary 2.5. Let $C$ be a finite dimensional purely inseparable field extension over $A$. If $A$ is the kernel of a set of higher derivations of $C$, then $C$ admits a p-basis over $A$.

Now let $x_{1}, \ldots, x_{m}$ be elements of $C$. It follows from

$$
\varphi_{D}\left(\prod_{i=1}^{m} x_{i}\right)=\prod_{i=1}^{m} \varphi_{D}\left(x_{i}\right)
$$

that

$$
D^{(l)}\left(x_{1} \cdots x_{m}\right)=\sum \prod_{i=1}^{m} D^{\left(\alpha_{i}\right)} x_{i}
$$

where the summation runs through all $\left(\alpha_{1}, \ldots, \alpha_{m}\right), \alpha_{i}$ nonnegative integers with $\sum_{i=1}^{m} \alpha_{i}=l$. Let $(l: m)$ denote the set of all these $m$-tuples and assume we are given $D_{1}^{\left(l_{1}\right)}, \ldots, D_{s}^{\left(l_{s}\right)}$ where $D_{i}$ are higher derivations on $C$. For any $\left(a_{1}, \ldots, a_{s}\right)$, $a_{i}=(\alpha(i, 1), \ldots, \alpha(i, m)) \in\left(l_{i}: m\right)$, set

$$
\left(a_{1}, \ldots, a_{s}\right)^{*}\left(x_{1}, \ldots, x_{m}\right)=\prod_{j=1}^{m}\left(\prod_{i=1}^{s} D_{i}^{\alpha(i, j)}\right) x_{j} .
$$

An induction on $s$ gives the following formula.

$$
D_{1}^{\left(l_{1}\right)} \cdots D_{s}^{\left(l_{s}\right)}\left(x_{1} \cdots x_{m}\right)=\sum\left(a_{1}, \cdots, a_{s}\right)^{*}\left(x_{1}, \ldots, x_{m}\right), \quad a_{i} \in\left(l_{i}: m\right) .
$$

LEMMA 2.6. Let $C$ be a local ring with $Q$ as its maximal ideal. Let $E$ be a $C$-algebra such that $E=C+J$ as a $C$-module direct sum for some finitely generated nilpotent ideal $J$ in $E$. Let $\pi: E=C+J \rightarrow J$ denote the second coordinate projection. Let $\mathfrak{g}$ be a set of higher derivations on $E$. Put $I=\{x \in J \mid \mu(x) \in Q+J$ for all $\mu \in \mathfrak{m}(\mathrm{g})\}$. 
Assume $\pi \mu(I) \subset I$ for all $\mu \in \mathfrak{m}(g)$. If $I \cap \mathfrak{F}^{i}(J) \subset \mathfrak{F}^{i}(Q J)$ for all $i$, then the product

$$
t_{1}^{q_{1}} \cdots t_{n}^{q_{n}} \neq 0(I)
$$

where $\left\{t_{1}, \ldots, t_{n}\right\}=\mathfrak{b}$ is a set of $p$-generators for $E=C+J, q_{i}=p^{e_{i}}-1, e_{i}=e\left(t_{i}\right)$ is the exponent of $t_{i}$ with respect to $C$.

Proof by contradiction. Let $m$ be the minimal integer such that for some integers $m_{i}, 0 \leqq m_{i} \leqq p^{e_{i}}-1$ and $\sum m_{i}=m$, we have

$$
z=t_{1}^{m_{1}} \cdots t_{n}^{m_{n}}=0(I) .
$$

We have $m>1$ because $I \subset Q J$. We claim that $m_{i}=0(p)$ for all $i=1, \ldots, n$. Assume this is not the case. Let $m_{1}, \ldots, m_{r}$ be nonzero modulo $p$ while $m_{i}=0(p)$ for all $i>r$. Write

$$
z_{i}=t_{i}^{m_{i}-1} \prod_{k \neq i} t_{k}^{m_{k}}, \quad i=1, \ldots, r
$$

The minimality of $m$ asserts that $z_{i}$ is nonzero modulo $I$. Let $l$ be the least integer such that for some $i, 1 \leqq i \leqq r, \mu\left(z_{i}\right)$ is a unit in $E$ for some $\mu \in \mathfrak{m}(\mathfrak{g})$ with degree $\mu$

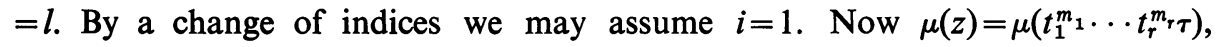
$\tau=\prod_{k>r} t_{k}^{m_{k}}$, can be expressed as a polynomial in $\mathfrak{b}$ with coefficients in $C$. We are going to show that the coefficient of $t_{1}$ in $\mu(z)$, which modulo $Q$ is unique, is a unit in $C$. This is not possible because $\pi \mu(z) \in I \subset Q J$. So $m_{i}$ must be zero modulo $p$ for all $i=1, \ldots, n$.

Put $\sigma=m_{1}+\cdots+m_{r}, \mu=D_{1}^{\left(l_{1}\right)} \cdots D_{s}^{\left(l_{s}\right)}$ and let

$$
a_{i}=\left(\alpha(i, 1,1), \ldots, \alpha\left(i, 1, m_{1}\right), \ldots, \alpha(i, r, 1), \ldots, \alpha\left(i, r, m_{r}\right), \alpha_{i}\right)
$$

be a general element of $\left(l_{i}: \sigma+1\right)$. Write

$$
\begin{aligned}
a & =\left(a_{1}, \ldots, a_{s}\right), \\
L(a, u, v) & =\text { the coefficient of } t_{1} \text { in } E_{u, v}=\left(\prod_{i=1}^{s} D_{i}^{(\alpha(i, u, v))}\right) t_{u}, \\
C(a, u, v) & =\text { the constant term of }\left(\prod_{(i, j) \neq(u, v)} E_{i, j}\right)\left(\prod_{i=1}^{s} D_{i}^{\left(\alpha_{i}\right)}\right) \tau .
\end{aligned}
$$

Given an $s$-tuple $b=\left(\beta_{1}, \ldots, \beta_{s}\right), 0 \leqq \beta_{i} \leqq l_{i}$, of integers, we denote by

$$
A(b, u, v) \text { the set }\left\{a \mid a_{i} \in\left(l_{i}: \sigma+1\right) \text { with } \alpha(i, u, v)=\beta_{i}\right\} \text {. }
$$

Since the coefficient of $t_{1}$ in $\left(\prod_{i=1}^{s} D_{i}^{\left(\alpha_{i}\right)}\right) \tau$ is zero modulo $Q$, the modulo $Q$ coefficient of $t_{1}$ in $\mu(z)=\left(D_{1}^{\left(l_{1}\right)} \cdots D_{s}^{\left(l_{s}\right)}\right)\left(t_{1}^{m_{1}} \cdots t_{r}^{m_{r} \tau}\right)$ is

$$
\sum_{u=1}^{r} \sum_{v=1}^{m_{u}} \sum_{a} C(a, u, v) L(a, u, v)=\sum_{u=1}^{r} \sum_{v=1}^{m_{u}} \sum_{b} \sum_{a \in A(b, u, v)} C(a, u, v) L(a, u, v) .
$$

We have the following cases 
(i) Not all of $\beta_{i}$ are zero. By the minimality of $l, \sum_{a \in A(b, u, v)} C(a, u, v)$ as the constant term of $\left(D_{1}^{\left(l_{1}-\beta_{1}\right)} \cdots D_{s}^{\left(l_{s}-\beta_{s}\right)}\right) z_{u}$ is zero modulo $Q$. Hence

$$
\sum_{a \in A(b, u, v)} C(a, u, v) L(a, u, v)
$$

is zero modulo $Q$.

(ii) $\beta_{i}=0$ for all $i=1, \ldots, s$ but $u \neq 1 . \sum_{a \in A(b, u, v)} C(a, u, v) L(a, u, v)$ is zero modulo $Q$ because $L(a, u, v)$ is.

(iii) $\beta_{i}=0$ for all $i=1, \ldots, s$ and $u=1$. Let $\mu\left(z_{1}\right)=\gamma+\nu$ with $\gamma \in C$ and $\nu \in J$. So

$$
\sum_{a \in A(b, 1, v)} C(a, 1, v) L(a, 1, v)=\gamma
$$

This shows $\pi \mu(z)=0$ modulo $I$ has a modulo $Q$ nonzero linear term $m_{1} \gamma t_{1}$ which is the desired contradiction.

Recall that the integer $e=e(J)$ is the least integer such that $\mathfrak{F}^{e+1}(J)=0$. From what we have shown we see that the lemma is true for $e=0$. Moreover, if the lemma is incorrect for some $e>0$, then it is also incorrect for $\mathfrak{F}(E)=\mathfrak{F}(C)+\mathfrak{F}(J)$ with $e(\mathfrak{F}(J))=e(J)-1$. An induction on $e$ completes the proof of the lemma.

3. The endomorphism ring. We begin with a slight rewording of the JacobsonBourbaki theorem. The proofs are adapted from Hochschild [1, Lemma 2.1 and Theorem 2.1].

Lemma 3.1. Let $C$ be a local ring with nilpotent maximal ideal $Q$. Let $\Omega$ be an $n<\infty$ dimensional free $C$-submodule of $\operatorname{Hom}_{z}(C, C)$ where $Z$ is the ring of all integers. Then there exist $c_{1}, \ldots, c_{n}$ in $C$ and a $C$-module basis $\omega_{1}, \ldots, \omega_{n}$ for $\Omega$ such that $\omega_{i}\left(c_{j}\right)=\delta_{i j}$.

Proof. Let $T_{0,1}, \ldots, T_{0, n}$ be any $C$-module basis for $\Omega$. We first observe that $T_{0, i}(C) \notin Q$ for all $i=1, \ldots, n$. For if $e$ is the least integer such that $Q^{e}=0$, then from $T_{0, i}(C) \subset Q$ we get $u T_{0, i}=0$ and hence $u=0$ for any $u$ in $Q^{e-1}$ which is absurd.

Now suppose we have already found $c_{1}, \ldots, c_{l}$ in $C$ and a $C$-module basis $T_{l, 1}, \ldots, T_{l, n}$ of $\Omega$ such that $T_{l, i}\left(c_{j}\right)=\delta_{i j}$, for $1 \leqq i \leqq n$ and $1 \leqq j \leqq l$. If $l<n$, there is an element $c_{l+1} \in C$ such that $T_{l, l+1}\left(c_{l+1}\right)$ is a unit in $C$. We set $T_{l+1, l+1}=$ $T_{l, l+1}\left(c_{l+1}\right)^{-1} T_{l, l+1}$, so that $T_{l+1, l+1}\left(c_{l+1}\right)=1$. For every $i \neq l+1$, we set $T_{l+1, i}=$ $T_{l, i}-T_{l, i}\left(c_{l+1}\right) T_{l+1, l+1}$. Then we have $T_{l+1, i}\left(c_{j}\right)=\delta_{i j}$, for $1 \leqq i \leqq n$ and $1 \leqq j \leqq l+1$, and that $T_{l+1, i}$ are still a $C$-module basis for $\Omega$. Proceeding in this fashion, starting from the case $l=0$, we finally obtain $c_{1}, \ldots, c_{n}$ in $C$ and $\omega_{i}=T_{n, i}$ which satisfy the requirements of the lemma.

LEMMA 3.2. Let $C$ be $a$ ring and $\Omega$ a (not necessarily commutative) subring of $\operatorname{Hom}_{z}(C, C)$. Assume that $\Omega$ is a free $C$-module based on $\omega_{1}, \ldots, \omega_{n}(n<\infty)$ such that for some $c_{1}, \ldots, c_{n}$ in $C, \omega_{i}\left(c_{j}\right)=\delta_{i j}$. Let $A$ denote the subring $\{c \in C \mid \omega(c x)$ $=c \omega(x)$ for all $x \in C$ and all $\omega$ in $\Omega\}$ of $C$. Then $C$ is a free $A$-module based on $c_{1}, \ldots, c_{n}$ and $\Omega=\operatorname{Hom}_{A}(C, C)$. 
Proof. Given $\omega$ in $\Omega$, if we write $\omega=\sum_{i=1}^{n} x_{i} \omega_{i}, x_{i} \in C$, then $x_{i}=\left(\sum_{j=1}^{n} x_{j} \omega_{j}\right)\left(c_{i}\right)$ $=\omega\left(c_{i}\right)$. In particular,

$$
\omega_{l}\left(x \omega_{j}\right)=\sum_{i=1}^{n}\left(\omega_{l}\left(x \omega_{j}\right)\right)\left(c_{i}\right) \omega_{i}=\omega_{l}(x) \omega_{j} \quad(x \in C) .
$$

So for any $c$ in $C, \omega_{l}(x) \omega_{j}(c)=\omega_{l}\left(x \omega_{j}(c)\right)$. It follows that $\omega_{j}(c) \in A$ for all $c \in C$ and $j=1, \ldots, n$. Now let $c \in C$ and write $c^{\prime}=c-\sum_{i=1}^{n} \omega_{i}(c) c_{i}$. We have $\omega_{j}\left(c^{\prime}\right)=0$ for all $j=1, \ldots, n$. So $c^{\prime}=0$ because $\omega_{j}$ form a basis for $\Omega$ which as a subring of $\operatorname{Hom}_{z}(C, C)$ contains the identity map on $C$. This shows $c=\sum_{i=1}^{n} \omega_{i}(c) c_{i}$ for all $c$ in $C$. If $\sum_{i=1}^{n} \alpha_{i} c_{i}=0, \alpha_{i} \in A$, then $\alpha_{i}=\omega_{i}\left(\sum_{j=1}^{n} \alpha_{j} c_{j}\right)=0$. Hence $c_{1}, \ldots, c_{n}$ form a basis for $C$ over $A$. Given any $f$ in $\operatorname{Hom}_{A}(C, C)$, we have $f=\sum_{i=1}^{n} f\left(c_{i}\right) \omega_{i}$. So $\Omega=\operatorname{Hom}_{A}(C, C)$. This completes the proof of the lemma.

THEOREM 3.3. Let $C$ be a local ring with nilpotent maximal ideal $Q$. Let $\mathrm{g}$ be a set of higher derivations on $C$ such that no ideal in $C$, except 0 and 1 , is stable under $\mathrm{g}$. Let $A$ denote the kernel of $\mathrm{g}$ and write $\Omega=C[\mathrm{~g}]$. If $\Omega$ is finitely generated as a $C$-module, then $\Omega=\operatorname{Hom}_{A}(C, C)$.

Proof. In view of Lemmas 3.1 and 3.2 above, it suffices to show that $\Omega$ is a finite dimensional free $C$-module. Let $\omega_{1}, \ldots, \omega_{n}$ be elements in $\mathfrak{m}(\mathfrak{g}) \subset \Omega$ such that the $\omega_{i}+Q \Omega$ form a basis for $\Omega / Q \Omega$ over $C / Q$. It follows from [5, p. 105, Corollaire 2] that $\omega_{1}, \ldots, \omega_{n}$ generate $\Omega$ as a $C$-module. If $\sum_{i=1}^{n} x_{i} \omega_{i}=0\left(x_{i} \in C\right)$, then $x_{i} \in Q$. Assume that not all the $x_{i}$ are zero. Let $\mu$ be an element in $\mathfrak{m}(\mathfrak{g})$ with minimal degree such that $\mu\left(x_{i}\right)$ is a unit in $C$ for some $i$ (Lemma 2.2). We have

$$
0=\mu\left(\sum_{j=1}^{n} x_{j} \omega_{j}\right) \equiv \sum_{j=1}^{n} \mu\left(x_{j}\right) \omega_{j} \text { modulo } Q \Omega
$$

which is a contradiction to the choice of $\omega_{j}$. This shows that $\Omega$ is a free $C$ module based on $\omega_{1}, \ldots, \omega_{n}$ as desired.

4. One derivation. Let $C$ be an algebra over a field $A$. Assume $C$ over $A$ admits a $p$-basis $\left\{t_{1}, \ldots, t_{r}\right\}$. We may assume the $t_{i}$ 's are units. For if $t_{i}$ is not a unit, it must be a nilpotent so can be replaced by $1+t_{i}$. Let $e_{i}$ be the exponent of $t_{i}$. By a change of indices we may assume $e_{1} \geqq \cdots \geqq e_{r}$. Let $D=\left\{D^{(1)}, \ldots, D^{(\rho-1)}\right\}$, $\rho=p^{e_{1}}$, be the higher derivation on $C$ corresponding to the $A$-algebra homomorphism

$$
\begin{aligned}
\varphi_{D}: C & \rightarrow C[z] /\left(z^{\rho}\right), \\
t_{1} & \rightarrow t_{1}+z, \\
t_{i+1} & \rightarrow t_{i+1}+\gamma_{i+1} z^{q_{i+1}},
\end{aligned}
$$

where $\gamma_{i+1}=\prod_{l \leqq i} t_{l}^{-1}, q_{i+1}=p^{e_{1}-e_{i+1}}$. We have the following

THEOREM 4.1. With notations as above,

$$
C[D]=\operatorname{Hom}_{A}(C, C) .
$$


Proof. The assertion is obviously true for $r=1$. When $r=1$ the following statement $(\mathrm{H})$ is also true.

(H) Given $a_{\lambda}$ in $A, 0<\lambda<p^{e_{r}}$, if there exists $x \in C$ such that

$$
\begin{aligned}
D^{\left(\lambda q_{r}\right)} x & =a_{\lambda} \gamma_{r+1}^{\lambda}, & & 0<\lambda<p^{e_{r},} \\
D^{(l)} x & =0, & & l \neq 0\left(q_{r}\right),
\end{aligned}
$$

then $x \in A\left[t_{r}\right]$ and $a_{\lambda}=0$ for all $\lambda$.

We are going to establish the following chain of implications:

(E) and (H) for all $r<s \Rightarrow(\mathrm{H})$ for $r=s \Rightarrow$ (E) for $r=s$.

Write

$$
x=\sum_{i=0}^{n-1} x_{i} t_{s}^{i}, \quad\left(n=p^{e_{s}}, x_{i} \in A\left[t_{1}, \ldots, t_{s-1}\right]\right)
$$

We have, for all $l>0$,

(1)

$$
\begin{aligned}
D^{(l)} x & =\sum_{i=0}^{n-1} D^{(l)}\left(x_{i} t_{s}^{i}\right) \\
& =\sum_{i=0}^{n-1} \sum_{\lambda}\left(D^{\left(l-\lambda q_{s}\right)} x_{i}\right) D^{\left(\lambda a_{s}\right)} t_{s}^{i} \\
& =\sum_{i=0}^{n-1} \sum_{\lambda}\left(\begin{array}{l}
i \\
\lambda
\end{array}\right) \gamma_{s}^{\lambda} t_{s}^{i-\lambda}\left(D^{\left(l-\lambda q_{s}\right)} x_{i}\right) \\
& =\sum_{j=0}^{n-1} t_{s}^{j} \sum_{i \geqq j}\left(\begin{array}{c}
i \\
i-j
\end{array}\right) \gamma_{s}^{i-j}\left(D^{\left(l-[i-j] q_{s}\right)} x_{i}\right) .
\end{aligned}
$$

Taking into account the assumption placed on $x$ in the statement $(\mathrm{H})$, we get for $l \neq 0\left(q_{s}\right)$,

$$
\sum_{i \geqq j}\left(\begin{array}{c}
i \\
i-j
\end{array}\right) \gamma_{s}^{i-j} D^{\left(l-[i-j] q_{s}\right)} x_{i}=0, \quad 0 \leqq j \leqq n-1
$$

In particular for $j=n-1$, we get

$$
D^{(l)} x_{n-1}=0
$$

for all $l \neq 0\left(q_{s}\right)$. Putting $j=n-2$ in (2) and taking into account (3) we get $D^{(l)} x_{n-2}$ $=0$ for all $l \neq 0\left(q_{s}\right)$. Hence

$$
D^{(l)} x_{i}=0
$$

for all $i$ and all $l \neq 0\left(q_{s}\right)$. Now put $l=\lambda q_{s}(\lambda \neq 0)$ in (1). From (H) we get

$$
a_{\lambda} \gamma_{s+1}^{\lambda}=\sum_{j=0}^{n-1} t_{s}^{j} \sum_{i \geqq j}\left(\begin{array}{c}
i \\
i-j
\end{array}\right) \gamma_{s}^{i-j} D^{\left([\lambda-i+j] a_{s}\right)} x_{i}
$$


So

$$
\begin{array}{rlrl}
\sum_{i \geqq j}\left(\begin{array}{c}
i \\
i-j
\end{array}\right) \gamma_{s}^{i-j} D^{\left([\lambda-i+j] q_{s}\right)} x_{i} & =a_{\lambda} \theta_{s} \gamma_{s}^{\lambda}, \quad j=n-\lambda, \\
& =0, & j \neq n-\lambda,
\end{array}
$$

where $\theta_{s}=\left(t_{s}^{\text {pes }}\right)^{-1}$. In particular

$$
D^{\left(q_{s}\right)} x_{n-1}=a_{1} \theta_{s} \gamma_{s} ; \quad D^{\left(\lambda q_{s}\right)} x_{n-1}=0 \quad(\lambda \neq 1) .
$$

By induction hypothesis we get $a_{1}=0$. So $D^{(l)} x_{n-1}=0$ for all $l \neq 0$. Applying the induction hypothesis again, we get $x_{n-1} \in A$.

Now assume $a_{i}=0, x_{n-i} \in A$ for all $1 \leqq i<k$. So

$$
\begin{array}{ll}
D^{\left(\lambda q_{s}\right)} x_{n-k}=0 & \text { for } \lambda>k \\
D^{\left(k q_{s}\right)} x_{n-k}=a_{k} \theta_{s} \gamma_{s}^{k} & \\
D^{\left(\lambda q_{s}\right)} x_{n-k}=-\left(\begin{array}{c}
n-k+\lambda \\
\lambda
\end{array}\right) \gamma_{s}^{\lambda} x_{n-k+\lambda} & \text { for } 1 \leqq \lambda<k .
\end{array}
$$

The induction hypothesis asserts that $a_{k}=0, D^{(l)} x_{n-k}=0$ for all $l>0$. So $x_{n-k}$ is also in $A$. This shows (H) is correct for $r=s$. In particular the kernel of $D$ is contained in $A\left[t_{s}\right]$. We claim that kernel $D$ is exactly $A$.

Let $x=\sum_{i=0}^{l} x_{i} t_{s}^{i}, x_{i} \in A, x_{l} \neq 0$, be an element of kernel $D$ with $l$ minimal. If $l$ is greater than zero, then

$$
D^{\left(l q_{s}\right)} x=\sum_{i=0}^{l} x_{i} D^{\left(l q_{s}\right)} t_{s}^{i}=x_{l} D^{\left(l q_{s}\right)} t_{s}^{l}=x_{l} \gamma_{s}^{l}
$$

is not zero because $\gamma_{s}^{l}$ is a unit; hence a contradiction.

We now contend that kernel $D=A$ implies $\operatorname{Hom}_{A}(C, C)=C[D]$. Let $M$ be the set of all monomials $t_{1}^{u_{1}} \cdots t_{s}^{u_{s}}, 0 \leqq u_{i}<p^{e_{i}}$. A lexicographic order may be imposed on $M$ as follows: $t_{1}^{u_{1}} \cdots t_{s}^{u_{s}}<t_{1}^{v_{1}} \cdots t_{s}^{v_{s}}$ if there is a $k$ such that $u_{k}<v_{k}$ and $u_{l}=v_{l}$ for all $l>k$. Given $f=\sum f_{u_{1}, \ldots, u_{s}} t_{1}^{u_{1}} \cdots t_{s}^{u_{s}}, f_{u_{1}, \ldots, u_{s}} \in A$, we denote by $O(f)$ the smallest element of $M$ such that $t_{1}^{u_{1}} \cdots t_{s}^{u_{s}} \leqq O(f)$ whenever $f_{u_{1}, \ldots, u_{s}}$ is not zero. We would like to show that given $x \neq 0$ in $C$ there is some $\mu \in \mathfrak{m}(D)$ such that $\mu(x)$ is a unit in $C$. Assume this is not the case. Let $f=\sum f_{u_{1}, \ldots, u_{s}} t_{1}^{u_{1}} \cdots t_{s}^{u_{s}}, f_{u_{1}, \ldots, u_{s}} \in A$, be a nonzero element in $C$ with the least $O(f)$ such that $\mu(f)$ is not a unit for any $\mu \in \mathfrak{m}(D)$. Since $0\left(D^{(l)} \zeta\right)<\zeta$ for all $l>0$ and $\zeta \neq 1$ in $M, f$ must belong to kernel $D$ which is the field $A$. But $f$ is not a unit so must be zero, hence a contradiction. This shows that no ideal in $C$, except 0 and 1 , is stable under $D$ (Lemma 2.2). It follows from Theorem 3.3 that $C[D]=\operatorname{Hom}_{A}(C, C)$.

\section{REFERENCES}

0. L. R. Harper, On differentiably simple algebras, Trans. Amer. Math. Soc. 100 (1961), 63-72. MR 24 \#A116.

1. G. Hochschild, Double vector spaces over division rings, Amer. J. Math. 71 (1949), 443-460. MR 10, 676. 
2. M. Sweedler, Structure of inseparable extensions, Ann. of Math. (2) 87 (1968), 401-410. MR 36 \#6391.

3. M. Weisfeld, Purely inseparable extensions and higher derivations, Trans. Amer. Math. Soc. 116 (1965), 435-449. MR 33 \#122.

4. Shuen Yuan, Differentiably simple rings of prime characteristic, Duke Math. J. 31 (1964), 623-630. MR 29 \#4772.

5. N. Bourbaki, Algèbre commutative. Chapitres I, II, Actualités Sci. Indust., no. 1290, Hermann, Paris, 1961. MR 36 \#146.

State University of New York at Buffalo,

AMHERST, New York 14226 